\title{
Acoso escolar en instituciones educativas de la ciudad de Huancayo en el 2011
}

\author{
Bullying in educational institutions of Huancayo city in 2011
}

Mildred Ávila Miñan', Sara Becerra Flores', Jeannette Vásquez Vega ${ }^{3}$, Sofía Becerra Flores ${ }^{4}$ Universidad Nacional Federico Villarreal

\section{RESUMEN}

Objetivos: Determinar la incidencia de acoso escolar en la ciudad de Huancayo identificando sus formas más recurrentes, índice de actores, atribución causal que éstos realizan y la situación sobre cómo y dónde se produce el maltrato. Métodos: De tipo exploratorio - descriptivo, enfoque cuantitativo y diseño no experimental, ex post facto y de corte transversal exigió la construcción y validación por criterio de jueces del cuestionario de incidencia de acoso escolar en una muestra de 256 escolares de 12 a 16 años (137 hombres y 119 mujeres) seleccionadas aleatoriamente, arrojando una confiabilidad de 0,68 según el Alfa de Crombach. Resultados: Se revela que el $100 \%$ de la población ha sufrido algún tipo de acoso, siendo el acoso psicológico el de mayor prevalencia, los hombres son más victimizados que las mujeres a nivel verbal y ellas son más víctimas de acoso físico. El acoso de los hombres es mixto, las mujeres emplean más el social. El $77 \%$ son observadores pasivos, el $27 \%$ restante hace algo para parar la situación de maltrato, el $21 \%$ afirma que este se da en los salones de clase, en la cancha de deportes o alrededor del colegio. Los agresores acosan porque los provocaron, las victimas dicen ser agredidas para molestarlas o por broma y los observadores acosan para molestar o divertir al grupo. Conclusiones: El acoso escolar es multicausal, de incidencia alarmante que se manifiesta bajo la forma de acoso psicológico donde 8 de 10 estudiantes han sido víctimas, agresores $u$ observadores, principalmente varones.

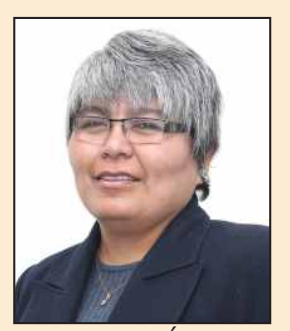

Mildred Ávila

Palabras clave: Acoso escolar, instituciones educativas, Huancayo.

1 Lic. en Psicología. Coordinadora del Dpto. Académico de Psicología de la Universidad Nacional Federico Villarreal.

2 Mg. en Psicología. Jefe de la Of. Grados y Títulos de la Facultad de Psicología de la Universidad Nacional Federico Villarreal

3 Lic. en Psicología. Jefe de Comunicaciones de la Facultad de Psicología de la Universidad Nacional Federico Villarreal.

4 Lic. en Psicología. 


\section{ABSTRACT}

Objectives: Determine the bullying incidence in Huancayo city identifying their more recurrent forms, the actors rate, the causal attribution that it realize and the situation about how and where the abuse occurs. Methods: Exploratory - descriptive type, quantitative research and not experimental design, ex post facto and transversal cross required for the construction and validation by judges criteria of the bullying incidences questionnaire in a sample of 256 scholars from 12 to 16 years old (137 men and 119 women) aleatory selected, giving a confidence of 0,68 according to Crombach's Alfa. Results: Has been revealed that $100 \%$ of the population has suffered some kind of bullying been of the major prevailing the psychological harassment, men are more victimized than women in a verbal form and women are victims of physical harassment. Men bullying is mixed, women use more a social form. The $77 \%$ are passive observers, the remaining $23 \%$ do something to stop the situation of abuse, and $21 \%$ confirms that this occurs in the classroom, at the sport field or around the school. Aggressors say they practice bullying because they were incited, victims say they are aggrieved to molest them or like a joke and observers practice bullying to molest or to distract the group. Conclusions: Bullying is multi causal with an alarming incidence expressed by psychological harassment where 8 of every 10 students have been victims, aggressors or observers, principally men.

Key words: Bullying, educational institutions, Huancayo.

\section{INTRODUCCIÓN}

Esta investigación se sitúa en un contexto global en el cual el fenómeno de la violencia escolar ha cobrado gran relevancia, amplificado en parte por la cobertura mediática de la prensa hacia diversos hechos de victimización escolar que han tenido dramáticas consecuencias. Independientemente de las razones que hayan confluido para poner este tema en boga, creemos importante tomar en serio la oportunidad que el interés nacional e internacional sobre la violencia en las escuelas, le da a la investigación social; asimismo, es importante porque nos da información acerca de este fenómeno en una ciudad del interior de nuestro país, tan cercano y a la vez tan lejano de nuestra capital.

No basta con reaccionar a sus manifestaciones explícitas interviniendo directamente sobre ellas; sino que, debemos comprender el fenómeno en su complejidad y a partir de allí, desarrollar formas preventivas realistas y eficaces, antes que sea "demasiado tarde".

Olweus, señala que un estudiante sufre de acoso escolar o es victimizado cuando es expuesto en forma reiterada y a lo largo del tiempo a acciones negativas por parte de uno o más estudiantes (1).

Nuestra investigación busca aportar al estudio de la violencia escolar situando el análisis en las condiciones psicológicas de la violencia dentro de la escuela en la ciudad de Huancayo, contribuyendo de esta manera a la generación de propuestas de prevención e intervención en violencia, que en el largo plazo, derivarían en la recuperación de los niveles de autoestima en la población escolar y por ende mejores niveles de desempeño en ese ámbito, la reducción del índice de deserción escolar por acoso, el fortalecimiento de factores protectores de conductas riesgos como consumo de drogas y alcohol, y la reducción del índice de suicidios y la prevención de la violencia estructural. En una dimensión más amplia, vinculada al desarrollo social, estaríamos contribuyendo en la construcción de ciudadanía y aportando en la generación de políticas públicas desde el ámbito académico en una perspectiva de responsabilidad social universitaria. 
Según el Informe Mundial sobre la Violencia y la Salud (2), América Latina es la región que sufre el mayor impacto de la violencia en el mundo, con una alta tasa promedio de homicidio de jóvenes entre 15 y 29 años, como manifestación más extrema, constituyéndose en la población más afectada por todo tipo de violencia (abuso físico, sexual, verbal, emocional y abandono) lo que limita a la vez, sus posibilidades de tener un desarrollo sano.

El mismo estudio cifra las víctimas españolas de "bullying" entre 11 y 18 años en $24,8 \%$ y afirma que el acoso y la violencia escolar se distribuyen de un modo muy desigual a lo largo del sistema educativo. Así, el porcentaje de víctimas en tercero de primaria asciende al $43,6 \%$ y desciende hasta un $10 \%$ en cuarto de Enseñanza Secundaria Obligatoria (ESO). Esta tendencia se replica en el estudio hecho por el Defensor del Pueblo (3) quien además halló que los alumnos entre 13 y 14 años son los que más sufren y ejercen violencia verbal y de exclusión.

García (4) observó que la presencia de violencia mixta (física y psicológica), en España, sólo se daba en la escuela, donde la amenaza entre compañeros tenía una frecuencia muy elevada (todos los días), aportando condiciones de alta inseguridad dentro de ella. En África, Gairín (5), encontró la amenaza como la forma de acoso de mayor frecuencia, siendo en algunos casos mayores incluso que el robo y el golpe entre alumnos.

Lo hallado por el Defensor del Pueblo en España (6) es muy similar, pues las agresiones verbales por parte de otros compañeros aparecen como la modalidad más frecuente afectando al $33 \%$ de los estudiantes. Este porcentaje se reduce a $20 \%$ si se trata de otro tipo de agresiones como las dirigidas a sus propiedades, alcanzando entre $9 \%$ y $14 \%$ en la modalidad de aislamiento permanente (sus compañeros los ignoran o no los dejan participar en nada). Las amenazas para amedrentar son sufridas por el $8 \%$ de la población y las agresiones físicas cerca del $5 \%$.
Monjas y Avilés (7), exploraron condiciones ambientales y grupales del bullying y la atribución causal de los participantes. Las auto-percepciones del alumnado muestran que las formas de maltrato varían conforme se avanza en edad, siendo la exclusión social y la ridiculización las formas propias del final de la etapa educativa. Previamente Avilés (8) identificó que el 5,9\% del alumnado señala intimidar sistemáticamente a sus compañeros y el $5,7 \%$ se reconoce como víctima sistemática; es decir, practican o reciben acoso "bastantes veces", "casi todos los días" o"casi siempre".

A nivel de Lima Metropolitana, Becerra, Flores y Vásquez (9) encontraron que el $45 \%$ de la población evaluada es agredida y de ella, el $67 \%$ es víctima de maltrato psicológico, no existiendo diferencias entre sexos. Las víctimas se sienten tristes, bajan sus notas, no duerme bien o están nerviosos, ocasionándoles sentimientos de rabia y odio hacia los demás, lo que trae como consecuencia el faltar a clases o fugar de casa.

En el mismo año, 2008, Oliveros y colb. (10) en un estudio transversal con 916 escolares, encontraron $47 \%$ de incidencia de bullying, llamando la atención que 1 de 4 maestros y padres de familia no reaccionan ni protegen a las víctimas permitiendo que este acoso continúe. La investigación revela también, el uso de la TIC's para acosar y las amenazas con navaja y arma de fuego que alcanzan cifras cercanas al 10\%, con una similar incidencia en el consumo de PBC, marihuana o terokal.

El acoso escolar es una forma característica y extrema de violencia escolar, es una especie de tortura, metódica y sistemática, en la que el agresor sume a la víctima, a menudo con el silencio, la indiferencia o la complicidad de otros compañeros. Se caracteriza por una reiteración encaminada a conseguir la intimidación de la víctima, que trae como consecuencia una serie de secuelas psicológicas que aterrizan en la afectación de la autoestima y la ruptura de su proyecto escolar. En casos más graves, puede acarrear pensamientos suicidas e 
incluso su materialización.

La víctima de acoso escolar suele generar, a mediano plazo, cuadros de estrés postraumático que van a dar lugar a cambios permanentes en su comportamiento, potenciando su vulnerabilidad ante otras formas de acoso y violencia como el maltrato doméstico y mobbing cuando llegan a la adultez. Pero los acosadores también sufren problemas y tienen más probabilidades de experimentar ansiedad y depresión, al mismo tiempo que afrontan mayor riesgo de suicidio que aquellos que no se involucran en la dinámica de acoso escolar.

En nuestra realidad local, los casos de acoso escolar vienen incrementándose en el campo y la ciudad, creando la necesidad de una investigación diagnóstica que nos permita establecer el punto de partida, así nos planteamos conocer ícuál es la incidencia de acoso escolar en Huancayo?

Nuestro objetivo principal fue determinar la incidencia de acoso escolar, para lo cual se contruyó y validó un "Cuestionario de Maltrato" en la población en estudio. Dicho instrumento nos permitió identificar la incidencia del fenómeno del maltrato y sus formas más recurrentes de acoso escolar; la incidencia de agresores, víctimas y observadores; y la situación sobre cómo y dónde se produce el maltrato, siempre considerando la variable sexo. Adicionalmente, identificamos la atribución causal que señalan los actores según el rol que desempeñan: víctimas, agresores $U$ observadores.

Respecto al origen de la violencia, creemos que es multicausal; sin embargo, las investigaciones impulsadas por organismos mundiales, identifican que las necesidades económicas y la desigualdad social promueven tanto el acoso escolar como otras formas de acoso en países en vías de desarrollo. A nivel de países desarrollados, señalan que los niveles crecientes de pobreza, desigualdad y exclusión social cumplen una función importante en la violencia en las escuelas.
A pesar de su alta incidencia y de ser ya un problema social, todavía hay muchas resistencias a aceptarlo y abordarlo, no desde los alumnos, sino desde los padres de familia y directivos de los centros educativos, principalmente particulares. El temor a encontrar índices de acoso entre sus estudiantes, lo que repercutiría en su imagen corporativa, es una de las limitaciones con que nos encontramos al momento de aplicar el cuestionario; motivo por el cual, no revelamos la identidad de las instituciones educativas de la ciudad de Huancayo.

\section{MATERIAL Y MÉTODOS}

La matrícula de $1^{\circ}$ a $5^{\circ}$ de secundaria en el año lectivo 2011 en los 61 colegios nacionales y particulares de la ciudad de Huancayo alcanza a 13375 escolares entre hombres y mujeres de 11 a 19 años. Usando la Tabla de Fisher - Arkin Colton, con un margen de error del 5\% y considerando como criterios de inclusión la edad ( 12 a 16 años) y la asistencia regular a clases, definimos el tamaño muestral en 364 escolares (202 hombres y 162 mujeres) que fueron seleccionados probabilísticamente y de manera polietápica. Seleccionamos al azar 7 colegios con un total de 3695 alumnos y determinamos por afijación proporcional el tamaño muestral para cada uno, lo mismo que para la proporción por sexo, dado que implican estratos de diferente tamaño.

Debido a que no se pudieron levantar los datos en algunos colegios con los que se había coordinado, por motivos propios de las instituciones, pese a que en un principio nos dieron el visto bueno, la muestra quedó reducida a 256 estudiantes. 
Tabla № 1: Distribución de la población y tamaño muestral.

\begin{tabular}{|c|c|c|c|c|c|c|}
\hline \multirow{2}{*}{ Colegios } & \multicolumn{3}{|c|}{ Población } & \multicolumn{3}{|c|}{ Muestra } \\
\hline & Total & Hombres & Mujeres & Total & Hombres & Mujeres \\
\hline Colegio 1 (privado) & 308 & 160 & 148 & 25 & 13 & 12 \\
\hline Colegio 2 (privado) & 335 & 200 & 135 & 27 & 17 & 10 \\
\hline Colegio 3 (público) & 1332 & 729 & 603 & 147 & 80 & 67 \\
\hline Colegio 4 (privado) & 729 & 396 & 333 & 73 & 40 & 33 \\
\hline Colegio 5 (privado) & 398 & 221 & 177 & 44 & 24 & 20 \\
\hline Colegio 6 (privado) & 283 & 179 & 104 & 23 & 14 & 9 \\
\hline Colegio 7 (público) & 310 & 168 & 142 & 25 & 14 & 11 \\
\hline Total & 3695 & 2053 & 1642 & 364 & 202 & 162 \\
\hline
\end{tabular}

\section{RESULTADOS}

El instrumento logró una confiabilidad de 0,68 según el Alfa de Crombach. Su aplicación revela que el $100 \%$ de la muestra ha observado algún tipo de acoso en el colegio donde estudia y manifiesta la prevalencia del maltrato físico en sus diferentes expresiones. Sin embargo, cuando evaluamos las cuatro formas básicas de maltrato, los resultados reflejan que es el psicológico el de mayor incidencia, seguido del maltrato verbal y físico.

Al hacer el análisis según sexo, encontramos que son los varones $(47,3 \%)$ quienes sufren mayor acoso de sus pares, principalmente de tipo psicológico y verbal. Lo curioso es encontrar que la agresión física entre niñas es más alta $(10,4 \%)$ que en varones $(9,6 \%)$. Al evaluar a los agresores, 8 de 10 han desempeñado ese rol, siendo mayor el porcentaje de varones (47\%) que el de mujeres (35\%). En cuanto al tipo de agresión, prevalece la psicológica en ambos sexos. Y con relación a los observadores, encontramos que 7 de cada 10 han presenciado alguna forma de acoso, siendo la misma proporción en varones que en mujeres. Pero lo que llama la atención es que el acoso se practique, principalmente, en el aula de clases $(21,1 \%)$, donde hay presencia docente. Otros espacios de acoso se dan alrededor del colegio $(19,8 \%)$, la cancha de deportes $(18,2 \%)$ y los patios
$(16,4 \%)$, con mayor incidencia.

La muestra evaluada coincide en que las conductas de acoso son multicausales destacando, en orden decreciente, el hacerlo por molestar, porque son mejores que los demás, porque cada uno tiene sus motivos para hacerlo y por divertir al grupo. En estos resultados llama la atención que la atribución causal de la conducta de acoso no varía mucho entre varones y mujeres desde el rol de observadores, haciéndonos pensar que el acoso es una conducta validada en su contexto de interacción. No muy diferentes son los resultados desde las víctimas, quienes señalan que sus compañeros los acosan simplemente por molestar $(15,3 \%)$ o por bromear $(14,8 \%)$, minimizando la acción. Pero, sí llama la atención el porcentaje de víctimas que señalan como causa del acoso, el ser diferente de los demás $(14,2 \%)$ o peor aún, ser ellos los causantes del acoso $(13,9 \%)$, justificando la acción.

Desde los agresores, el 27,2\% de la población manifiesta que agrede al otro porque éste lo provocó. Todas las demás causas se dispersan, sin dejar de llamar la atención el $8,1 \%$ que revela su conducta agresiva por miedo a que lo agredan a él, y el 2,7\% que refiere su agresión sin saber por qué lo hace. 


\begin{tabular}{lccc}
\multicolumn{1}{c}{ Causas } & Hombre & Mujer & Total \\
\hline No lo sé & 21 & 18 & 39 \\
Se meten con ellos & 23 & 26 & 49 \\
Son más fuertes & 45 & 28 & 73 \\
Por broma & 43 & 37 & 80 \\
Por molestar & 72 & 78 & 150 \\
Por ser mejores & 68 & 65 & 133 \\
Divierten al grupo & 59 & 46 & 105 \\
Es normal & 24 & 22 & 46 \\
Tienen sus motivos & 69 & 48 & 117 \\
Para pertenecer al grupo & 29 & 29 & 58 \\
Así lo educaron & 35 & 48 & 83 \\
Así lo tratan en su casa & 34 & 37 & 71 \\
Es propio de la edad & 29 & 19 & 48 \\
\hline
\end{tabular}

\section{DISCUSIÓN}

La aproximación al tema de violencia escolar no resulta tan distante si se tiene en cuenta que en la sociedad siempre se han dado comportamientos de esta naturaleza, sólo cambian los métodos y escenarios. Desde Paco Yunque hasta hoy, el maltrato en las escuelas y entre pares ha estado presente. La evolución de las conductas de agresión va de la mano con aquello que la sociedad acepta como "adecuado", así, las primeras agresiones se expresan en insultos, apodos, discriminación y se han ido agravando hasta llegar al empleo de amenazas, rumores, descrédito del otro, discriminación racial, violencia física con uso de armas y en casos extremos, agresiones severas que llevan desde la depresión hasta la muerte por suicidio $u$ homicidio, replicando hasta cierto punto la realidad de América Latina según el Informe Mundial sobre la Violencia y Salud (2).

La incidencia de "bullying" hallada, es alarmante si consideramos que el $100 \%$ de la población refiere haber observado alguna forma de acoso en sus escuelas, duplicando la incidencia hallada en Lima por Becerra, Flores y Vásquez (9) en el que sólo el $45 \%$ de la población reportaba haber presenciado. De las diferentes formas de acoso presenciadas, el acoso físico se reporta con mayor incidencia, seguido del acoso psicológico y verbal. Ello tiene sentido si consideramos que las agresiones físicas son visibles, a diferencia de las otras formas, que se asumen, muchas veces, como parte del patrón de comunicación en la convivencia. Sin embargo, cuando consultamos sobre las formas de acoso experimentadas, es el acoso psicológico el de mayor incidencia, llamando la atención el que no exista una diferencia significativa entre los sexos, semejante a lo hallado por Becerra, Flores y Vásquez (2008).

En la dinámica del bullying se articulan tres actores: agresor, victima y observador, cada uno con rol propio que determina su actuar. En Huancayo, 8 de cada 10 escolares, reconoce haber sido víctima de algún tipo de acoso, y con sorpresa encontramos que los hombres son más victimizados que las mujeres, tanto psicológica como verbalmente. Estos resultados podrían explicarse desde los roles de género y señalar que existe mayor agresión entre varones que entre mujeres, lo cual coincide con lo evidenciado en la realidad social y es reportado por los medios de prensa.

En el análisis del tipo de acoso a las víctimas 
según sexo, encontramos que es el acoso psicológico desde los varones el que se reporta con mayor frecuencia, ellos agreden psicológicamente más a sus víctimas en comparación con las mujeres, y en general, este tipo de acoso supera largamente a los otros. Sin embargo, consideramos importante señalar que desde las mujeres hay una mayor incidencia, aunque no significativa, de agresión física hacia sus víctimas con relación a los varones, lo que nos lleva a especular que las agresiones psicológicas entre mujeres se acompañan, muchas veces, de agresiones físicas. O también, que este tipo de agresión no es vista como tal desde lo varones, porque las conductas rudas y "juego de manos" es aceptado como natural en la interacción con sus pares del mismo sexo. De hecho, los varones emplean más la violencia física, mientras que las chicas utilizan más la agresión verbal o de exclusión o esparcen rumores maliciosos.

Al tratar de establecer la incidencia de agresores por sexo, resultan siendo los varones los que más agreden (8 de 10), similar a la percepción de las víctimas, donde 8 de cada 10 estudiantes refieren haber sido víctima de acoso. Estas cifras producen alarma pues estamos frente a conductas agresivas que podrían estar instalándose como "adecuadas" en la dinámica de las relaciones sociales y familiares.

Otro punto importante en la práctica del bullying es la respuesta de los observadores frente al acoso en sus diferentes manifestaciones. El $77 \%$ sólo observa y no hace nada, convirtiéndose en cómplices. El otro $23 \%$ hizo algo como cortar la situación, ayudar a la víctima, informar a algún adulto o hablar con quién agrede.

Estas diferentes formas de acoso se da en varios lugares dentro del colegio, llamando nuestra atención que es el aula de clase donde hay mayor frecuencia $(21,1 \%)$, el único espacio de todos los consultados, donde el profesor está presente de manera permanente, llamando nuestra atención sobre las formas de regulación del acoso y la percepción de autoridad. Los otros espacios como el patio, los baños, los pasillos, la cancha deportiva y los alrededores del colegio, son también escenarios de acoso,pero en menor dimensión. Estos resultados coinciden con los hallados por Hernández y Casares (11) que afirman que la violencia puede darse en cualquier espacio del colegio. Sin embargo, en las aulas es donde siempre "suele surgir la chispa", el detonante del problema. Igual que Avilés (2002), que encontró que los actos de intimidación se producen en su mayoría, lejos del alcance de adultos y más dentro que fuera del recinto escolar, teniendo como espacios preferentes la clase sin profesor y los recreos sin vigilancia.

Estas prácticas de acoso tienen un origen, diferente desde la perspectiva de cada uno de los actores. Los observadores atribuyen el acoso al sólo hecho de molestar, sin mayor diferenciación entre varones y mujeres; o lo atribuyen al deseo de creerse mejores que sus compañeros; otros refieren que cada acosador tendrá sus motivos o lo hacen por el simple hecho de divertir al grupo. Estas respuestas podrían estar reflejando el poco interés de los escolares por hacer algo que detenga las conductas acosadoras, encontrando, por el contrario, una justificación de ellas.

Con menor incidencia, los observadores refieren que el acoso es normal, que es propio de la edad, que así los educaron o que así los tratan en casa. Estas apreciaciones refuerzan la percepción de "normalidad" de las conductas acosadoras, restándoles relevancia como problema de conducta al interior de las escuelas. Pero también debemos tomar en cuenta el rol de los observadores, quienes siendo conscientes del acoso prefieren mantenerse al margen en un instinto de auto protección, pues de otra manera podrían ser ellos las víctimas.

Desde las víctimas, si bien atribuyen el acoso al hecho de que los quieren molestar o por bromear, justificando de alguna manera las agresiones recibidas, nos preocupa que lo atribuyan a características de ellos mismos, 
como: "me acosan porque soy diferente", "soy débil", porque "no respondo" o "porque yo mismo lo provoqué", dando indicadores de baja autoestima, de sentirse merecedores del maltrato.

Es importante resaltar que en la respuesta "no respondo" hay una diferenciación por sexo, siendo las mujeres quienes atribuyen el maltrato a su incapacidad para responder, pero no necesariamente, se consideran débiles en comparación con sus pares varones. Otra respuesta que nos inquieta es "yo los provoqué" también de mayor incidencia en las mujeres, justificando la agresión desde el otro. Estas respuestas podrían estar reflejando una cadena de violencia alrededor de los estudiantes, afectando sus desempeños académico y social, y alterando la valoración de lo adecuado, resultando ser, la violencia, un patrón normal de conducta. Y es allí dónde surge la pregunta si la cultura social, donde todavía prevalece el machismo, tiene alguna influencia en la atribución de la conducta de agresión.

Desde la óptica de los agresores, el acoso se justifica principalmente en la provocación que hacen los otros, minimizando su actuación. Este resultado se torna relevante si lo analizamos desde la perspectiva del locus de control, asumiendo que estos jóvenes atribuyen la causalidad de la conducta a factores externos y no a decisiones propias. Esta percepción sobre la responsabilidad de los actos podría constituirse en predictor del desempeño futuro en el ámbito social, familiar y laboral.

Otras causas del acoso se atribuyen a la autopercepción de superioridad que no relaciona necesariamente con la autoestima. Los agresores señalan que acosan a sus compañeros porque son mejores, por divertirse o simplemente porque le dan ganas de molestar, transgrediendo los límites del respeto por el otro. También podemos observar agresión como exclusión, "los acoso porque son débiles", "no son del grupo", "no es mi amigo" o "son diferentes". Menor incidencia respecto de las causas pero no menos relevantes, son "porque así me tratan" o "a mí me lo hacen", transitando sus roles entre víctima y agresor. Un porcentaje menor atribuye su conducta a que es propio de la edad, por tanto, no sancionable; y otros, al miedo, podríamos decir que, como mecanismo de protección frente a la agresión de los otros.

Concluyendo, podemos afirmar que la mayor incidencia de acoso se da bajo la forma de acoso psicológico, y la atribución causal de la conducta acosadora descansa también en aspectos psicológicos relacionados con el autoconcepto, sentido de pertenencia al grupo y necesidades de seguridad, desde los diferentes actores. 


\section{REFERENCIAS BIBLIOGRÁFICAS}

1. Olweus D. Conductas de acoso y amenaza entre escolares. Madrid: Morata; 1998.

2. OPS/OMS. Ruta crítica del acoso escolar: análisis y resultados de investigación. Panamá: OPS; 2003.

3. Defensor del Pueblo. Informe de Defensor del Pueblo Español, Violencia Escolar: el maltrato entre iguales en la educación secundaria obligatoria. Madrid: Aran;2007.

4. García A. Investigaciones realizadas en España sobre Violencia Escolar. Revista Electrónica Interuniversitaria de Formación del Profesorado. 2002; 5(1).

5. Gairín J. Organización Escolar e Innovación Didáctica. Madrid: UNED; 1989.

6. Defensoría del Pueblo. Informe del Defensor del Pueblo sobre violencia escolar. Madrid: Aran. 1999.

7. Monjas M, Avilés J. Estudio de incidencia de la intimidación y el maltrato entre iguales en la educación secundaria obligatoria mediante el cuestionario CIMEI. Rev. Anales de Psicología. $2005 ; 21(1): 27-41$.

8. Avilés J. El acoso entre iguales (bullying) agresores/as y víctimas en la escuela entre iguales. Valencia: Almar. 2002.

9. Becerra S, Flores E, Vásquez E. Acoso escolar en Lima. (Trabajo de investígación). Lima: UNFV; 2008.

10. Oliveros M, Figueroa L, Mayorga G, Cano B, Quispe Y, Barrientos A. Violencia escolar (bullying) en colegios estatales de primaria en el Perú. Rev. Pediatr. 2008, 61 (4): 215-220.

11. Hernández T, Casares E. Aportaciones teórico prácticas para el conocimiento de actitudes violentas en el ámbito escolar. Pamplona: Instituto Navarro de la mujer. 2002.

12.Álvarez L, Álvarez D, González Castro P, Núñez J, Gonzáles J. Evaluación de los comportamientos violentos en los centros educativos. Psicothema, 2006; 18, (4), 686 - 695.

13. Debarbieux. Comprehensive Approaches to School Safety and Security: an international view. 2002; París: OECD.

14.Fullan M, Hardreaves A. What's Worth Fighting for in Your School?. New York: Open University Press; 1992.

15.Plata, C.; Riveros, M. y Moreno, J. Autoestima y empatía en adolescentes observadores, agresores y víctimas del bullying en un colegio del municipio de Chía. Psychologia: Avances de la disciplina. 2010; Vol. 4. N².99-112.

16. Shaw M. Promoting Safety in Schools: International Experience an Action. New York: International Centre for the Prevention of Crime: 2002.

\section{Correo electrónico:}

mavila@hotmail.com 